Proceedings of the 2011 Winter Simulation Conference

S. Jain, R.R. Creasey, J. Himmelspach, K.P. White, and M. Fu, eds.

\title{
AN APPLICATION OF DISCRETE-EVENT SIMULATION TO AN OUTPATIENT HEALTHCARE CLINIC WITH BATCH ARRIVALS
}

\author{
Michael Findlay \\ The University of Oklahoma \\ 202 W. Boyd St., Room 124 \\ Norman, OK 73019-0631, USA
}

\author{
Hank Grant \\ The University of Oklahoma \\ 202 W. Boyd St., Room 124 \\ Norman, OK 73019-0631, USA
}

\begin{abstract}
An application of discrete-event simulation is performed on an unique outpatient primary care clinic serving a military population at Fort Sill, OK. Access to the clinic is on an exclusively walk-in basis. Arrivals occur primarily in batches; arrival times and batch sizes are stochastic in nature. The nursing and medical staffs available each day also follow a stochastic process. The arrival process is characterized through discrete distributions. Several alternatives are modeled to examine procedural changes that may result in improved patient flow and provider utilization. A hybrid appointment/walk-in model was determined to have the best promise for improvement, but the possible benefits it may yield do not seem to justify the costs of its implementation in this setting. Possible applications of the hybrid model to other facilities such as urgent care facilities are discussed.
\end{abstract}

\section{INTRODUCTION}

Many of us are aware of the increased attention access to health care has received and the reports on struggles of balancing costs and resources with the quality and availability of care and services. The military is not immune to these challenges either. In providing healthcare to its service-members, the military must concern itself not only with quality of health and life issues, but also with readiness. To that end, the military devotes tremendous resources.

One such example can be seen at the U.S. Army's Sergeant Bleak Troop Medical Clinic (TMC) in Fort Sill, OK. There, the TMC provides outpatient primary care services to Soldiers in basic combat training with a dedicated staff made up of 9 healthcare providers (physicians and physician's assistants), 10 nurses and 12 medics. The clinic is supported by an administrative staff and also has an in-house pharmacy, basic laboratory, and X-ray imaging capability. Moreover, the clinic is equipped with a treatment area where minor procedures and treatments can be performed.

Depending on the time of the year, there may be fewer than 1,000 Soldiers in basic combat training or, in the case of summer, the population of basic combat training Soldiers may swell to well over 6,000 as the Army experiences a large influx of new enlistees. The staff of the TMC calls this time of the year the "summer surge" and it should come as no surprise that it is then that they see the highest volume of patients.

Soldiers have access to the clinic during two sick call periods - a two-hour period both in the morning and afternoon - on an exclusively walk-in basis. The number of Soldiers arriving on sick call and the acuity of their complaints vary greatly from day to day. During the summer surge, this uncertainty often stresses the clinic's systems and its staff. The TMC leadership speculates that there may be better ways to operate the TMC. This research is an effort to provide the TMC with an analysis of their current operational policies and potential alternatives via discrete-event simulation (DES). A further aim of this research is to expand the body of knowledge on the application of DES to outpatient healthcare settings, es- 


\section{Findlay and Grant}

pecially those with uncertain arrival rates. Many examples of DES applications have been studied, but none have considered a situation like the TMC that provides acute primary care with exclusively walk-in access to that care.

\section{LITERATURE REVIEW \& BACKGROUND}

Numerous studies have been performed that apply the use of DES to healthcare settings. Mustafee, Katsaliaki, and Taylor (2010) produced a systematic survey of the healthcare simulation in the literature. They found that no fewer than 51 papers published between 1970 and 2007 had used DES in an application to healthcare (Mustafee, Katsaliaki, and Taylor 2010). Jacobson, Hall, and Swisher (2006) also offer a thorough review of such efforts over the last 40 years.

Jacobson, Hall, and Swisher (2006) state, "High quality, efficient patient flow is a function of high volume patient throughput, low patient waiting times, short total visit times, and low levels of staff overtime coupled with the maintenance of reasonable staff utilization rates and low physician idle times." In fact, there are plentiful examples in the literature of researchers using these measures, especially patient times and physician utilization, to describe system performance. Among these are the papers by Edwards et al. (1994) and Rising, Baron, and Averill (1973).

Emergency departments are characterized by unpredictable patient arrivals and a broad spectrum of complaint acuity (Jacobson, Hall, and Swisher 2006). Emergency department staffs are well aware of the stresses these stochastic processes cause. Since demand at emergency departments is increasing and patient conditions can be life-threatening, endeavors to mitigate these stresses in emergency departments are the reasons why they are among the most popular applications to improve patient flow. Work done by Takakuwa and Shiozaki (2004) and by Sinreich and Marmor (2004) are examples of such research. Whereas the Takakuwa and Shiozaki (2004) work described a model suited specifically for their application, Sinreich and Marmor (2004) proposed a model that could be applied much more generically.

Research involving applications to emergency departments are of particular relevance to a TMC application, because they have similar arrival processes. Both operate exclusively on a walk-in basis with random patient arrivals. As we will discuss later, the TMC arrival process is not completely unpredictable. Neither is the case with most emergency departments. Analysis of arrival patterns will sometimes show trends according to the day of the week or time of day. Such consideration to these patterns is witnessed in the research done by Garcia et al. (1995), Mahapatra et al. (2003), and by Medeiros, Swenson, and DeFlitch (2008).

The new ideas on patient flow in emergency departments presented in the literature produce great results and they certainly merit consideration for adaptation to the design of alternatives for patient flow in the TMC. However, the patients arriving to the TMC do not present nearly the spectrum of complaint acuity as seen in emergency departments. The TMC's role is to provide primary care to its patients. Even though there is a triage station at the TMC, its function is mostly limited to an administrative onechecking for complete documentation and ensuring a relatively uniform assignment of patients to the providers available that day. Patients in the TMC are not routinely prioritized according to their complaint. Even when a patient is deemed acute enough to justify being moved ahead in the queue, these occurrences are so rare that access to care at the TMC can comfortably be characterized as first come, first serve. The TMC differs from emergency departments in another respect as well. In general, emergency departments are continuously open. The TMC has set hours - it opens and closes every day and is completely closed on Sundays.

Clearly, applications to emergency departments do not provide a complete basis of knowledge for understanding the TMC system. Fortunately, much research has been completed on applications to outpatient clinics, including primary clinics, with the intent of improving patient flow.

Clinics operating under an appointment system also concern themselves with the problems of noshows, which have a significant impact on the variability in patient arrivals. Even a clinic providing specialized care such as the cancer clinic studied by Santibáñez et al. (2009), experienced a no-show rate of

$6 \%$. Appointment no-shows are of such concern, research has even been done to identify what factors 


\section{Findlay and Grant}

can accurately predict the likelihood a particular appointment will be honored. Lee et al. (2005) developed such a model with $80 \%$ accuracy.

Research by Giachetti et al. (2005) discusses how a clinic with a high average no-show rate attempted to compensate by "over-booking" appointments. Sometimes, this over-booking policy led to longer wait times and patient dissatisfaction. The researchers proposed as a solution a policy called Open Access, in which a majority of appointment slots were deliberately kept open so that same-day appointments could then be scheduled, as there was good reason to believe that patients receiving an appointment on the day of the request were much less apt to no-show (Giachetti et al. 2005).

Research performed at an urgent care facility with walk-in arrivals shows how a proposed change to doctor scheduling to better serve demand can result in significantly reduced patient waiting times (Tan, Gubaras, and Phojanamongkolkij 2002). The Rising, Baron, and Averill (1973) study also shows the effects of physician scheduling changes on waiting times. Another study of an outpatient clinic shows how changing the flow of patients from a serial queueing system to a "quasi-parallel" system improved performance (Edwards et al. 1994). At those facilities discussed in the literature that accept walk-in arrivals, the patients were subsequently examined by staff on a first-available basis (Tan, Gubaras, and Phojanamongkolkij 2002; Rising, Baron, and Averill 1973). In the TMC, patients are assigned early on in the process to a particular provider with some regard in keeping these assignments uniform in quantity across the providers available on that day.

None of the outpatient examples discussed so far involve situations with exclusively walk-in patients. The clinic studied in the paper by Rising, Baron, and Averill (1973), however, did allow walk-in patients in addition to its scheduled appointments. What makes the TMC system unique among those studied in the literature is also the most challenging to characterize: the arrival process. As mentioned earlier, arrivals occur to the TMC on a walk-in basis. The arrival times are stochastic and each arrival actually represents a batch of patients. The sizes of these batches are also stochastic. Therefore, multivariate data must be collected on the arrival process. However, it cannot be assumed that these variates are independent of each other. In fact, observations at the TMC quite definitively showed that the batch size was greatly influenced by the time of the arrival. Law (2007) discusses that it can very difficult to estimate a multivariate distribution for collected data. He proposes some distributions that have been shown to be useful in simulations that explicitly specify the correlation structure and joint distributions, but concedes this may be too rigid for all cases. It was decided in this research to use an empirical distribution of the batch sizes and use the correlation structure observed directly. Law (2007) cautions that the risk of using an empirical distribution is that it may not completely represent all of the values possible. The relative abundance of arrival data available, though, makes the risk in this case acceptable.

Not only is the aim of this research project to have its results applied to the TMC in particular, but also to other settings in general. No studies were found on applications to facilities that combine the features of exclusively walk-in access and outpatient health services, such as the TMC. Dozens of such facilities exist elsewhere in the military and no doubt they would be interested in the results presented here. These results should also be applicable to the TMC's civilian cousin: the urgent care facility. Since the prevalence of urgent care facilities is on the rise (Hendrick 2008) as more and more people discard traditional doctor offices and hospitals in favor of them, the TMC research may prove to have broad implications.

\section{METHODOLOGY}

\subsection{General System Description}

Arrivals may occur to the TMC during two two-hour periods, one in the morning and one in the afternoon Monday through Friday. Patients arrive only during the morning period on Saturdays. Arrivals occur on an exclusively walk-in basis. The TMC has no foreknowledge of who will be seeking care on any given day; let alone what their individual complaints may be. Arrivals occur primarily in batches as the patients are transported by their training unit to the clinic en masse. 


\section{Findlay and Grant}

When a patient enters the TMC, they must first pass through a triage station. The medic positioned at the triage station assigns patients to a specific provider. Patients are subsequently directed to the check-in area. The triage medic also takes care to ensure self-care patients - those with minor complaints and only requiring over-the-counter treatments - are routed directly to the pharmacy area. Self-care patients are not required to wait in the triage queue and they exit immediately after receiving service at the pharmacy.

At the check-in station patients are served by one of 3 clerks. Upon completion of the check-in service, the clerks direct patients to one of three particular screening areas according to the provider with whom they have been assigned by the triage medic. At the screening area, patients have their vital signs taken and are interviewed by a nurse or medic (nurses and medics are largely utilized interchangeably at the TMC). Upon conclusion of most of these interviews, the nurse or medic will next route the patient to their assigned provider's area. However, some of these interviews result in the nurse or medic invoking a protocol called Algorithm Directed Troop Medical Care (ADTMC).

Under ADTMC, the nurse or medic initiates care, some of which can be completed in the screening room. Other times, the nurse or medic may bring the patient to the treatment room for services that must be performed there. It is only after initiation of care under ADTMC that the nurse or medic consults with the patient's assigned provider. Sometimes, the consultation alone is sufficient, and the patient may not even have a traditional encounter with the provider in the examination room as the provider is satisfied the patient received the appropriate level of care. At the other extreme, the patient may require a procedure — such as a toenail removal — at which point the patient takes priority over all others already present in their provider's queue.

Patients routed to their assigned provider are served on a first come first serve basis. Upon conclusion of this encounter, patients may or may not require further treatment and ancillary services. Some patients are sent to the treatment room. The provider may also determine a procedure is necessary and perform this in the treatment room. Other patients are sent to the X-ray area. Others are sent to the pharmacy. And there is also a subset of patients whose care is complete with the conclusion of the provider encounter. These patients are sent directly to the check-out area.

The treatment room provides many services and items such as intravenous fluids, wound care, and blood draws. There are normally two nurses dedicated to providing these services in the treatment room to all the patients there at any given time. The treatment room does not follow a conventional queueing model for service where patients compete for the resources, nurses. Rather, the nurses perform tasks as needed. The nurses are free to attend to many patients and do not provide service to the patients sequentially. Following the treatment room, patients are routed either to the pharmacy or to the check-out station according to the orders of their provider.

The X-ray area follows a more conventional single server queueing model. Patients arrive and are served on a first come first serve basis. X-ray images are taken according to the providers' orders. Following service in the X-ray area, patients are routed to the check-out station.

The pharmacy is operated by a two-person team made up of a pharmacy technician and a pharmacist. Patients turn in their documents to the pharmacy technician and wait to be called by the pharmacist when their prescriptions have been filled. Patients proceed directly to the check-out station after receiving their prescriptions. The check-out station is in the same physical location as the check-in area and is served by the same clerks. Patients checking out from the clinic take priority over patients that are checking in. The check-out station serves to close out the patients' visit at the TMC.

One other rule governing the TMC's operation has a significant impact on patient flow. When the lunch break starts, and if there are still patients present in the TMC, those patients not actively receiving service are asked to leave and invited to return following the break. When these patients do return, they may immediately return to the queue they were in before lunch, although their rank in the queue may not have been preserved. Figure 1 provides a visual representation of the TMC system. This conceptual model determined what data were to be collected and also served as the basis for implementation. 


\section{Findlay and Grant}

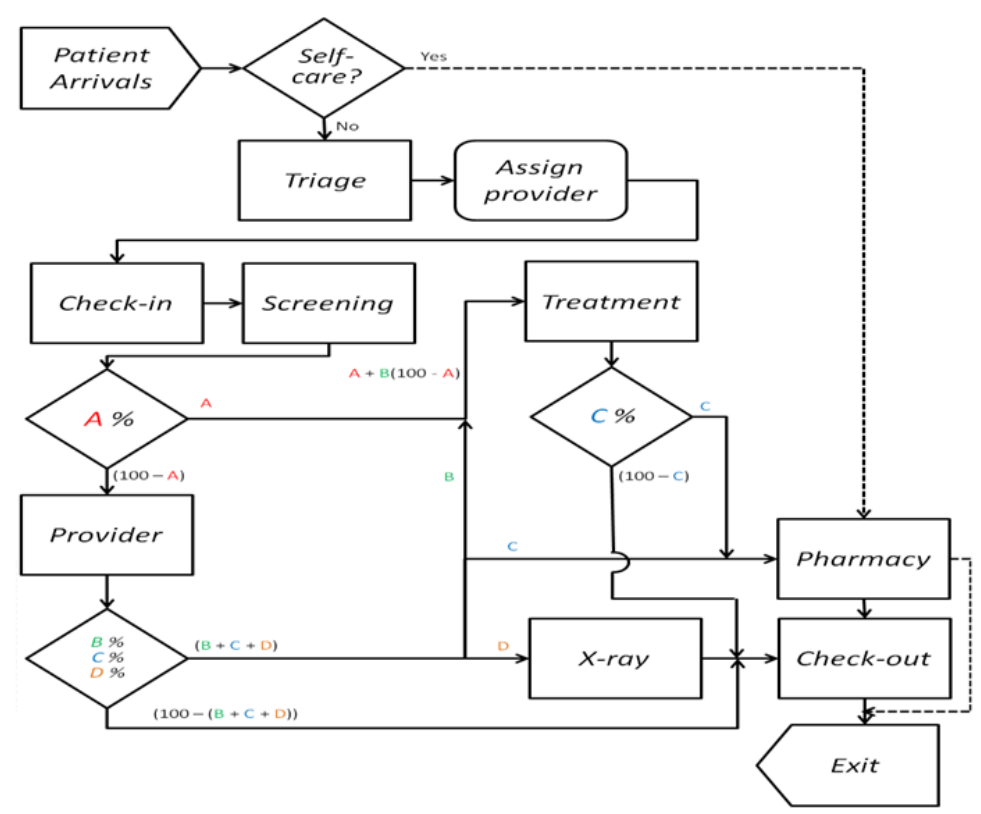

Figure 1: Conceptual model describing TMC patient flow. Variables A, B, C, \& D describe the likelihood patients require ancillary services.

\subsection{Modeling Assumptions}

A few assumptions were made; some due to limited data and others made in order to simplify modeling the system. First, not all patients arriving at the clinic represented an original visit for any one complaint. Often, patients were placed on "quarters", a means to exclude Soldiers from training and allow them to rest, and then re-evaluated the following day to determine if they were fit to return to duty. Others returned the day after their first visit in order for the provider to follow-up on results from a test ordered the previous day, such as from the lab or X-ray. It was assumed, then, that these follow-up patients were well represented in the arrival data and no effort was made to model patients requiring a follow-up visit.

There are a relatively large number of patients in the TMC system; and the presence or absence of any one patient has a negligible impact on the overall resource utilization in the TMC. Moreover, arrivals are generated by as many as a dozen or more sources (the training units) and the decisions leading to arrivals from those sources are independent of one another. For these reasons, it is fair to assume that the size of the arrival batches can be represented with a Poisson process.

Since the service time data on the TMC's processes were collected over many days - even weeks for some processes - and on different individual resources it was assumed that the collected service times are independent. Since there was also a lack of anecdotal evidence pointing to correlation of service times for adjacent processes and because of the large number of patients in the system, it was assumed any such correlations, if they did exist, would have a negligible effect on the overall system. Therefore, the threat of introducing bias into the model was perceived to be very low.

Even though it may have been possible to determine differences in the service times of individual providers, it was decided to ignore any such differences. This simplified the modeling effort in avoiding the need to track and reproduce the work schedules of individuals. This assumption also reduced the need to collect service time data for all providers, although an effort was made to collect at least a sample from a majority of the providers. Finally, treating the providers generically prevents a situation in which the providers may feel that they are being compared with one another. This rationale was also applied to the data collected for the nurses and the medics in the screening process. They were also considered to be a generic resource. The validity of these assumptions will be borne out further in the discussions on input analysis and model validation. 


\section{Findlay and Grant}

\subsection{Input Analysis}

Based on the conceptual model, data had to be collected on several distinct processes. These data were collected over the course of the summer of 2010. To the extent possible, data were used that were already being captured by the TMC. In the absence of reports, automatic or otherwise, summarizing the TMC's processes, data were collected through personal observation. For 13 days during the summer period, data were collected on the arrival process by recording the time of each arrival and the number of patients (or batch size) in that arrival. Care was taken to also note how self-care patients and patients interrupted by the lunch break were represented in the arrivals. 423 distinct observations were recorded in this fashion.

The check-in process, screening process, and provider encounters were also observed personally at various points over the summer, numbering 192, 100, and 227 observations, respectively. The service time of these processes were recorded for each patient observed. The patient's destination was also noted, when possible and appropriate, following service in these processes. Data were similarly collected on the triage, pharmacy, and check-out processes. 285 service times for the treatment room and 421 service times for X-ray area were obtained from their own records.

To characterize the arrival process, random distributions were fitted to the inter-arrival times data. Then, an empirical distribution was developed to represent the batch sizes of these arrivals. The same empirical distribution generated from aggregating the batch sizes from each of the 13 days arrival data was therefore used to model the arrival patterns for each day of the week. However, in terms of the absolute volume of arrivals, differences were noted based on the day of the week. The empirical distribution was scaled appropriately to account for the differences in patient volume amongst the days of the week. These scaling factors were developed by comparing the average daily patient census for each day of the week and also taking into account the relative weight each day of the week had in the original empirical distribution. Modeling the arrival process in this way allows for an empirical description of the correlation between batch size and arrival time. When an arrival is generated in the model according to the parameterized random distribution fitted to the inter-arrival data, the model will then take a random draw according to a Poisson distribution with mean value from the appropriate time interval of the empirical distributions shown in Figures 2 and 3. This result is scaled according to the factors in Table 1 and yields the batch size for the generated arrival.

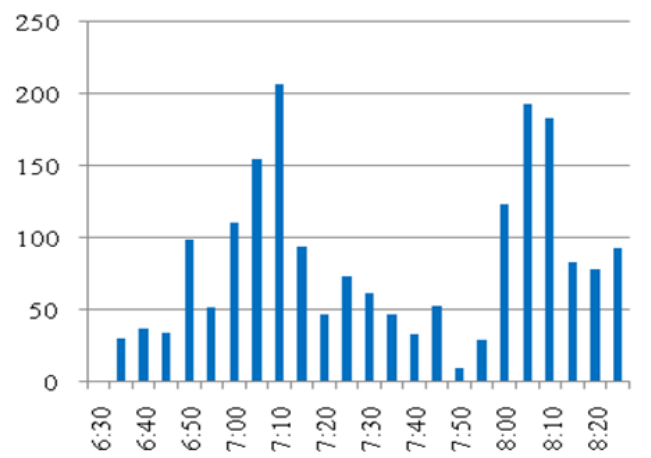

Figure 2: Batch sizes aggregated over 13 days for morning arrivals by 5 -minute intervals.

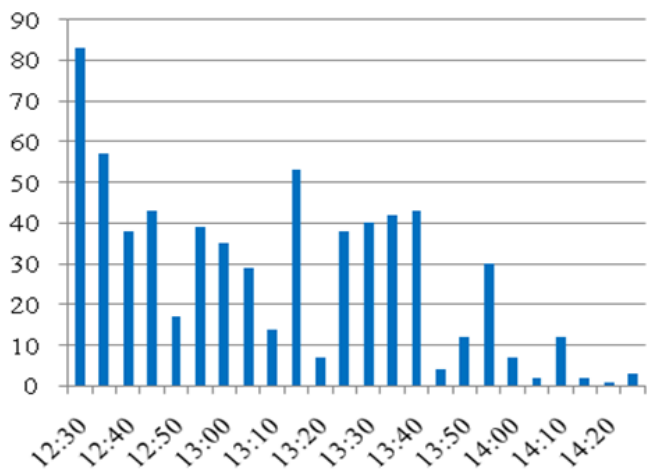

Figure 3: Batch sizes aggregated over 13 days for afternoon arrivals by 5 -minute intervals.

Table 1: Average daily arrivals are relative to the busiest day, Monday.

\begin{tabular}{l|cccccc} 
& $M$ & $T$ & $W$ & $T h$ & $F$ & $S a$ \\
\hline Normalized average arrivals & 1 & 0.77 & 0.71 & 0.65 & 0.78 & 0.27 \\
\hline Calculated scaling factors & 0.65 & 0.50 & 0.46 & 0.42 & 0.51 & 0.17
\end{tabular}




\section{Findlay and Grant}

Describing the service times for the various processes in the TMC system was much more straightforward. Parameterized random distributions were fitted using the Arena Input Analyzer (Kelton, Sadowski, and Sturrock 2007) on the observed data of each process, except for the triage station, pharmacy, and check-out station. The ratio of self-care patients to gross arrivals was calculated based on personal observations of the arrival process. To determine which portion of patients required ancillary services such as treatment, X-ray, or pharmacy during the visit, the total number of visits at those services was divided by the net arrivals of patients for those days the service data were available. No attempt was made to identify correlation amongst these ratios. Again, because of the large number of patients in the system, it was reasoned any impact attributable to any such correlations would be lost in the noise of the model. The number of providers and screening nurses and medics available on weekdays also vary.

The TMC maintains static staffing levels of 3 providers and 6 nurses/medics on Saturdays. TMC daily and weekly rollup reports were used to count the number of providers that worked each day. From this, discrete probability distributions were constructed to describe how many providers, nurses and medics may be on duty any given day. Those patients whose visits to the TMC were interrupted by the lunch break did not return to the TMC in a deterministic fashion. Some returned right as the clinic reopened for the afternoon, but others did not return until almost halfway through the afternoon arrival window. Based on observations of the arrival process it was determined that these interrupted patients should be modeled as having an additional delay in hours following lunch according to the $\operatorname{UNIF}(0,1)$ distribution.

\subsection{Validation of Base Model}

To validate the model implemented in Arena, several measures were examined. First, it was necessary to validate that the arrival logic was an appropriate description of the actual arrival process. To do so, $95 \%$ confidence intervals were constructed of both the observed and simulated average net arrival values and compared to see if there was statistical evidence of significant differences (Walpole et al. 2007). As Figure 4 illustrates, no statistically significant differences were found.

Next, the total time patients spent in the TMC system was evaluated. As patients were received at the triage station, the triage medic time stamped their paperwork. When these same patients checked out of the TMC, the clerks recorded the triage time stamp and the check out time - the difference being the time the patients had spent in the TMC. 610 observations were recorded in this fashion. An identical statistic was collected in the simulation. The electronic reports of the X-ray area made it an excellent candidate for collecting statistics on its queue. 85 observations from a week with arrival numbers similar to the average values were used for comparison. Again, there is no statistical evidence to suggest differences exist when comparing the actual system to the model. Validation results are shown in Figure 5.

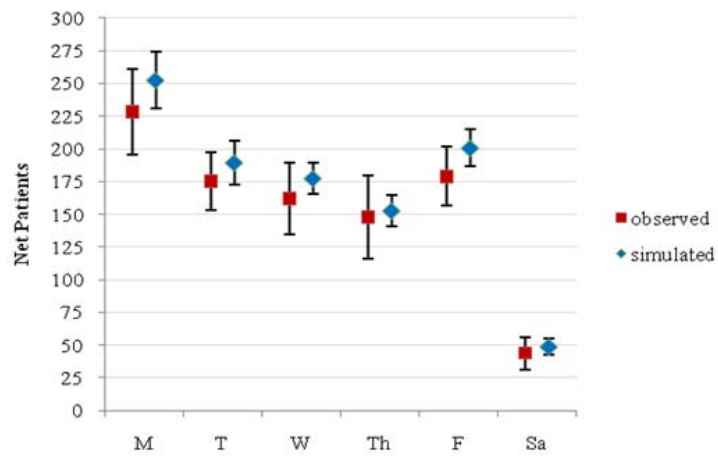

Figure 4: 95\% confidence intervals of average daily arrivals.

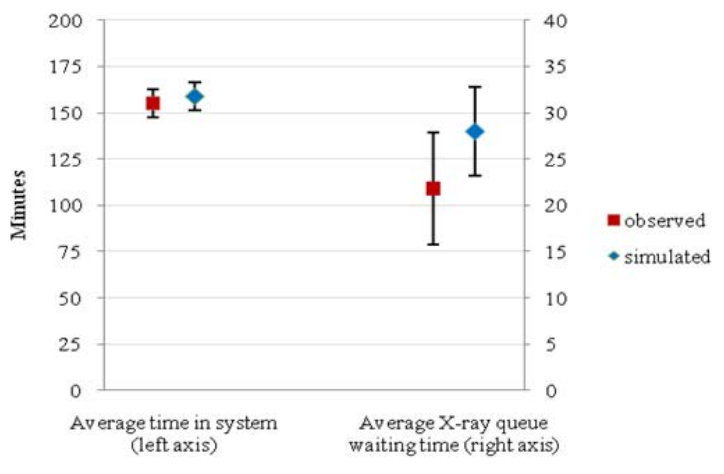

Figure 5: 95\% confidence intervals of selected validation statistics. 


\section{Findlay and Grant}

\subsection{Experimentation}

With a validated base model, attention turned to investigating several alternatives to current TMC operations. Alternatives were modeled in the Arena software environment. Each replication consisted of one week of TMC operations and a total of 30 replications were run for each alternative. To compare the various alternatives, three performance measures were identified. First, the average time in system (TIS) was used to gauge how the alternatives performed in terms of patient flow. Second, a modified provider utilization statistic was used to judge how efficiently the bottleneck resource of the TMC was being used. So that more accurate values of provider utilization could be obtained and to reduce the influence variation in patient census would have on the measure, provider utilization was not calculated across the entire replication. Instead, a provider utilization statistic was calculated only for the period from 6:30 to 3:00 daily for those providers on duty that day, taking in to account their breaks and lunch. These daily utilization results were then aggregated for the week from Monday through Friday. Third, the average numbers of patients interrupted by the lunch break daily were compared to assess performance in patient throughput.

Since Monday was the busiest day at the TMC by a wide margin, it was thought that ensuring that there was a certain minimum number of providers available on Monday may lead to increased performance. An alternative that guaranteed a minimum of seven providers, in contrast to five under the base model, was examined. Under the TMC's observed provider scheduling paradigm, they can on average expect 38 provider-days per week. The Saturday census is held static at three providers. To compensate for the increased minimum provider level on Monday, the probability distribution for Tuesday through Friday had to be adjusted accordingly to maintain 38 provider-days for the week.

Next, an alternative that eliminated most of the occasions when a patient would have their visit interrupted by the lunch break was examined. In this alternative model, the schedules of the providers, nurses, and medics were staggered such that these resources would always be available in the TMC. During the lunch break, directed queue jockeying was modeled to shift patients from one provider queue to another as their lunch schedules dictated in an attempt to minimize waiting times. Since the pharmacy and X-ray areas possess low-density resources, though, patients requiring these services over the original lunch break period still experienced an interruption to their visit. In these instances, the model logic for interruptions under this alternative proceeded as it did for the base model.

Two alternative models were developed to consider changes in queueing behavior. First, an alternative model using a queue common to all screening nurses/medics and a queue common to all providers was designed. As a result, patients were not assigned a specific provider at the triage station. This model maintained all other procedural logic found in the base model. There is a concern, however, that a model of this type may be impractical to actually implement in the TMC due to its physical layout. To address this concern, a model with parallel queues was developed. Instead of assigning patients to a specific provider and being screened by a set of nurses/medics according to this assignment, patients were instead routed first to the screening nurse/medic with the fewest patients waiting in their queue. Next, to be routed to a provider, the patient was put into the shortest provider's queue. The disadvantages of the common queue and shortest-queue assignment models are that they do not preserve continuity from patient to provider nor do they maintain TMC team integrity.

The implementation of an appointment system may encounter difficulties in being implemented; chief among these are decreased responsiveness and lack of an adequate transportation infrastructure. In case these difficulties were ever to be overcome, it was worth exploring what the system performance might look like if an appointment system was imposed upon the TMC. A model was developed that eliminated the walk-in arrival process for weekday operations (Saturday still employed an exclusively walk-in arrival process). Under the base model, the average number of weekly arrivals was roughly 1,100 patients. An appointment schedule was developed that brought 216 patients to the clinic daily.

Finally, a model that was a hybrid of an appointment and walk-in system was created. In this model, appointments were allocated such that they agreed with the provider census. The number of appointments available was determined by the provider census. It was assumed that patient demand for the TMC would remain the same as it was under the base conditions. Whatever average demand could not be absorbed by 


\section{Findlay and Grant}

the appointment structure was considered to be walk-in. In this way, patients not able to obtain an appointment on the day they desired care would be allowed access to the TMC on a walk-in basis during the two walk-in periods used in the base model. Patients with appointments were assigned a specific provider, whereas walk-in patients were routed to the providers with the shortest queues at the time. Again, just as it was in the pure appointment model, a $0 \%$ no-show rate was assumed.

\section{RESULTS AND ANALYSIS}

Three of the alternatives offer significant reductions for patient average TIS: the staggered lunch scheme, and both the pure and hybrid appointment models. These results should not be unexpected. Since the staggered lunch model reduces the waiting time patients incur on average by not interrupting as many over the lunch period, a reduced average TIS should naturally follow. The models involving appointments act to spread out the arrival times of patients, so again it is not that surprising that the average TIS would decrease. See Table 2.

Table 2: 95\% confidence intervals of selected performance measures.

\begin{tabular}{c|cc} 
& $\begin{array}{c}\text { Average TIS } \\
\text { (minutes) }\end{array}$ & $\begin{array}{c}\text { Average provider } \\
\text { utilization }\end{array}$ \\
\hline \hline Base & $(151.34,166.62)$ & $(0.564,0.604)$ \\
\hline Weighted Monday & $(148.61,164.71)$ & $(0.572,0.612)$ \\
\hline Staggered lunch & $(129.14,149.70)$ & $(0.591,0.651)$ \\
\hline Shortest queue & $(153.95,176.47)$ & $(0.600,0.660)$ \\
\hline Common queue & $(139.24,155.82)$ & $(0.567,0.607)$ \\
\hline Appointment & $(67.69,70.59)$ & $(0.476,0.496)$ \\
\hline Hybrid & $(106.31,117.27)$ & $(0.584,0.604)$
\end{tabular}

Analysis of patients interrupted by the lunch break gives insight to the dynamics of the TMC system. As Figure 6 demonstrates, there is a significant reduction in average interruptions on Monday in both the staggered lunch and appointment models.

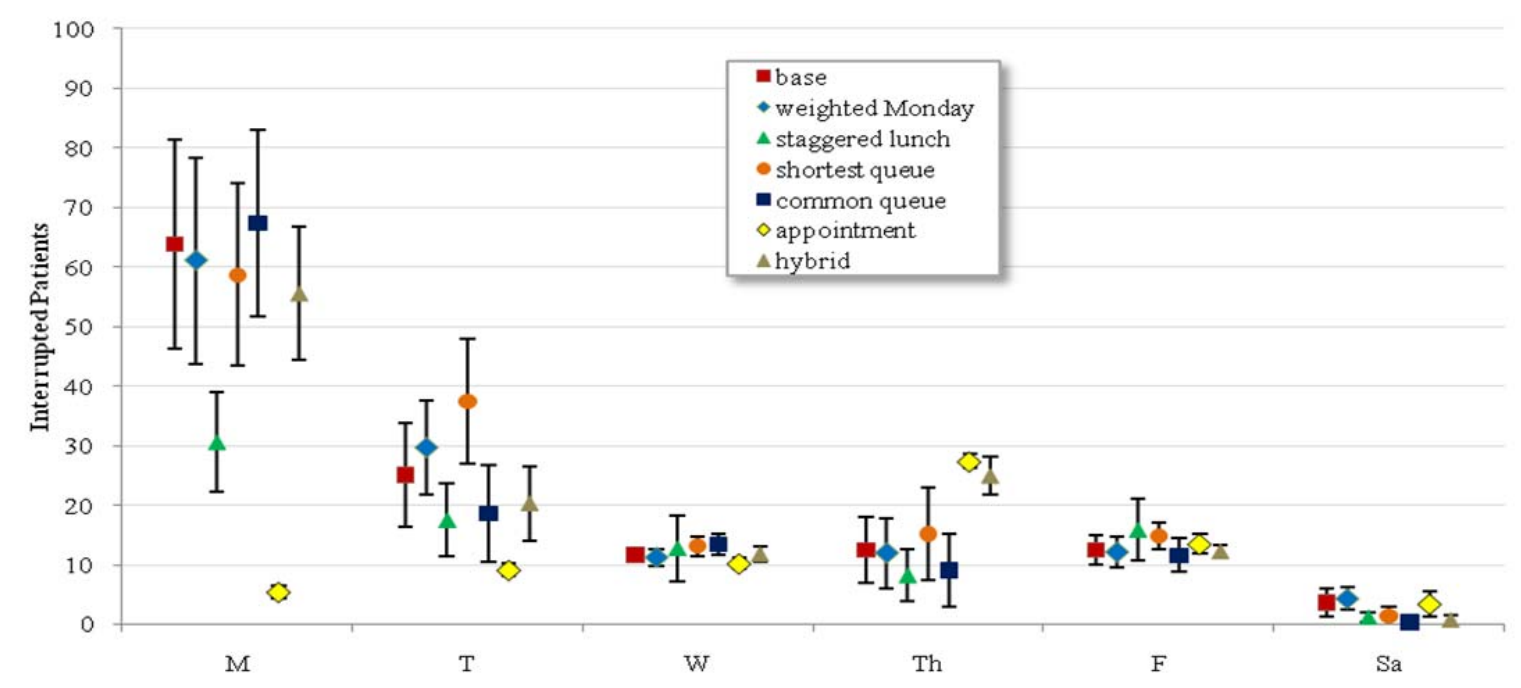

Figure 6: 95\% confidence intervals of average interruptions due to the lunch break.

Only the appointment model shows a significant reduction in average interruptions on Tuesday. Also, the interruption behavior of all the models across Tuesday through Friday is similar, which agrees 


\section{Findlay and Grant}

with their comparable patient volumes. Monday, in contrast, has a markedly higher average patient volume than the other days of the week. These results taken together lead to the conclusion that the primary source of patient interruptions on Monday is that providers are unable to exhaust their queues before lunch. Because Tuesday through Friday did not exhibit similar behavior, it would seem that the primary source of interruptions on those days is due to the queues in ancillary services, such as pharmacy and Xray, not being vacant at the start of the lunch break.

\section{CONCLUSIONS}

What, if any, of these alternatives should be recommended? Both the staggered lunch and hybrid models show improvements in average TIS with no decrease in average provider utilization, so they are likely candidates. The cost of implementing the staggered lunch alternative may not be worth the modest savings in average TIS. Introducing a staggered lunch schedule would create an item that would have to be vigilantly managed. Then, there is the question of lunch for patients that are not interrupted as they would be under current policies. In the basic training environment, skipping lunch is just not an option, so providing that lunch for patients in the TMC over the traditional lunch period adds a logistical burden to the training units. They would probably not agree that a staggered lunch model best serves their needs.

Even though there are modest gains in the average TIS for the staggered lunch and hybrid models, these models do not produce a significant change in weekly provider utilization. Though these alternatives do reduce the length of a TMC visit from the patient's perspective, it is not because the procedural changes result in delivering patients to the providers any more efficiently. In fact, the pure appointment model actually causes a significant reduction in provider utilization. It would seem that an appointment system, while minimizing the average TIS, results in a tradeoff in provider utilization.

The appointment component of the hybrid model represents an idealized situation. It is unimaginable that in practice $100 \%$ of the appointment slots would be filled, let alone honored. The objections that the training units would have to appointments still remain. Appointments, by definition, are not immediately responsive. They guarantee care at a future time. As it is now, there is immediate access to care, and using our average TIS statistic, the visit is complete just over 2.5 hours later. It is also hard to imagine the training units investing the resources required to transport Soldiers to individual appointments. At best, they would bring them all at once and Soldiers with appointments later on would simply wait on the benches positioned conveniently outside of the TMC's front entrance. It would be true, then, that those patients waiting outside until their appointment would, on average, have a shorter TIS, but they would not enjoy any actual time savings. At the other extreme, patients arriving all at once would simply be treated as walk-in arrivals, thereby relegating the appointment structure obsolete.

The average provider utilization rates-generally around $60 \%$ - may make it appear that the TMC system is inefficient. There is scheduled provider capacity that is idle in the system and this generally occurs right before and after lunch; as some days the morning arrival patients are exhausted before lunch and it also takes time for patients arriving after lunch to reach the providers. This conclusion is further corroborated by the fact that attempts to improve patient flow in the shortest queue and common queue models had no significant effect on performance. It appears that potential starving of the provider queues (the premise of constructing the alternative queue models) is not what drives the utilization rates. Instead, it is the periodicity of patient arrivals. As it has already been discussed, strategies for smoothing out the stream of patients are untenable. So, the TMC is left with little recourse to capitalize on the identified unused provider capacity.

Should this be viewed adversely? On very busy days, possibly exacerbated by a low provider census, the provider utilization rate can be as high as $98 \%$. In essence, the TMC uses provider capacity as a buffer against the uncertainty associated with variability in patient volume. It should also be said that even though these models report provider idle times, in actuality the providers are still gainfully employed. Providers can use the time they are not actively seeing patients to perform other administrative duties, follow-up on tests they ordered, consult with specialists, etc. In fact, many providers view this "idle" time as 


\section{Findlay and Grant}

an opportunity to catch up on the work they must put off when they experience a day with high patient volume.

What, then, of the attempt to weight capacity to the busiest day: Monday? In order for that attempt to show significant improvements, it would probably have to guarantee eight or even the maximum, nine, providers work on Monday, as opposed to the minimum of seven as modeled. That is probably not achievable as many of the factors - sick days, diversions to higher-priority missions, mandatory training, to name a few-contributing to the stochasticity of provider scheduling are beyond the TMC's ability to influence.

Through experience, the TMC has judged that having two walk-in periods at the start of both the preand post-lunch portions of the day results in the best throughput as can be obtained given external constraints. The training units prefer this arrangement as well, as they must only concern themselves with transporting Soldiers to and from the TMC at specified times of the day. When viewed in a broader context, the TMC system is actually quite remarkable. In an average week at the TMC during the "summer surge", volume is around 1,100 patients. Considering that there are an average of 7 providers on duty each day, this equates to each provider seeing more than 30 patients per day, making the TMC the busiest primary care facility in the entire Fort Sill medical system. Yet, they are able to accommodate patients on a same-day basis, on average in a little over 2.5 hours. The TMC performs as well as it probably can under its current operating procedures and any interventions that may result in modest benefits in some areas are more than outweighed by the cost of their implementation.

Should a facility like the TMC find itself under different circumstances, such as a location with a robust transportation infrastructure, or the ability to pool resources with other like facilities, then perhaps it would be wise to consider some of the alternatives tried here, such as the hybrid model. Such a model would most likely suit an urgent care facility very well as their patients are ostensibly able to provide their own means of transportation. It would also be worth further research to examine the effects a dynamic scheduling scheme would have on system performance. Although such a scheme could not be implemented at the TMC for practical reasons, it may suit other like systems in order to better match capacity against demand, so that such a large capacity buffer would not have to be maintained.

\section{REFERENCES}

Edwards, R. H., J. E. Clague, J. Barlow, M. Clarke, P. G. Reed, and R. Rada. 1994. "Operations Research Survey and Computer Simulation of Waiting Times in Two Medical Outpatient Clinic Structures." Health Care Analysis: HCA: Journal of Health Philosophy and Policy 2(2):164-169.

Garcia, M. L., M. A. Centeno, C. Rivera, and N. DeCario. 1995. "Reducing Time in an Emergency Room Via Fast-Track." In Proceedings of the 1995 Winter Simulation Conference, edited by C. Alexopoulos, K. Kang, W. R. Lilegdon, and D. Goldsman, 1077-1080. Piscataway, New Jersey: Institute of Electrical and Electronics Engineers, Inc.

Giachetti, R. E., E. A. Centeno, M. A. Centeno, and R. Sundaram. 2005. "Assessing the Viability of an Open Access Policy in an Outpatient Clinic: A Discrete-Event and Continuous Simulation Modeling Approach.” In Proceedings of the 2005 Winter Simulation Conference, edited by M. E. Kuhl, N. M. Steiger, F. B. Armstrong, and J. A. Joines, 2246-2255. Piscataway, New Jersey: Institute of Electrical and Electronics Engineers, Inc.

Hendrick, B. 2008. "'Doc in the box': Urgent Care Explosion." The Atlanta Journal-Constitution, June 29, 2008.

Jacobson, S. H., S. N. Hall, and J. R. Swisher. 2006. "Discrete-Event Simulation of Health Care Systems." In Patient Flow: Reducing Delay in Healthcare Delivery, edited by R. W. Hall, 211-252. New York: International Series in Operations Research and Management Science.

Kelton, W. D., R. P. Sadowski, and D. T. Sturrock. 2007. Simulation with Arena. $4^{\text {th }}$ ed. New York, NY: McGraw-Hill.

Law, A. 2007. Simulation Modeling and Analysis. $4^{\text {th }}$ ed. New York, NY: McGraw-Hill. 


\section{Findlay and Grant}

Lee, V. J., A. Earnest, M. I. Chen, B. Krishnan. 2005. "Predictors of Failed Attendances in a MultiSpecialty Outpatient Centre Using Electronic Databases.” BMC Health Services Research 5:51.

Mahapatra, S., C. P. Koelling, L. Patvivatsiri, B. Fraticelli, D. Eitel, and L. Grove. 2003. "Pairing Emergency Severity Index5-Level Triage Data With Computer Aided System Design to Improve Emergency Department Access and Throughput." In Proceedings of the 2003 Winter Simulation Conference, edited by S. Chick, P. J. Sánchez, D. Ferrin, and D. J. Morrice, 1917-1925. Piscataway, New Jersey: Institute of Electrical and Electronics Engineers, Inc.

Medeiros, D. J., E. Swenson, and C. DeFlitch. 2008. "Improving Patient Flow in a Hospital Emergency Department." In Proceedings of the 2008 Winter Simulation Conference, edited by S. J. Mason, R. R. Hill, L. Mönch, O. Rose, T. Jefferson, and J. W. Fowler, 1526-1531. Piscataway, New Jersey: Institute of Electrical and Electronics Engineers, Inc.

Mustafee, N., K. Katsaliaki, and S. Taylor. 2010. "Profiling Literature in Healthcare Simulation." Simulation 86(8/9):543-558.

Rising, E. J., R. Baron, and B. Averill. 1973. "A Systems Analysis of a University-Health-Service Outpatient Clinic." Operations Research 21(5):1030-1047.

Santibáñez, P., V. S. Chow, J. French, M. L. Puterman, S. Tyldesley. 2009. "Reducing Patient Wait Times and Improving Resource Utilization at British Columbia Cancer Agency's Ambulatory Care Unit Through Simulation." Health Care Management Science 12(4):392-407.

Sinreich, D., and Y. Marmor. 2004. "A Simple and Intuitive Simulation Tool for Analyzing Emergency Department Operations." In Proceedings of the 2004 Winter Simulation Conference, edited by R. G. Ingalls, M. D. Rossetti, J. S. Smith, and B. A. Peters, 1994-2002. Piscataway, New Jersey: Institute of Electrical and Electronics Engineers, Inc.

Takakuwa, S., and H. Shiozaki. 2004. "Functional Analysis for Operating Emergency Department of a General Hospital." In Proceedings of the 2004 Winter Simulation Conference, edited by R. G. Ingalls, M. D. Rossetti, J. S. Smith, and B. A. Peters, 2003-2011. Piscataway, New Jersey: Institute of Electrical and Electronics Engineers, Inc.

Tan, B. A., A. Gubaras, and N. Phojanamongkolkij. 2002. "Simulation Study of Dreyer Urgent Care Facility." In Proceedings of the 2002 Winter Simulation Conference, edited by E. Yücesan, C. -H. Chen, J. L. Snowdon, and J. M. Charnes, 1922-1927. Piscataway, New Jersey: Institute of Electrical and Electronics Engineers, Inc.

Walpole, R. E., R. H. Myers, S. L. Myers, and K. Ye. 2007. Probability \& Statistics for Engineers \& Scientists. $8^{\text {th }}$ ed. Upper Saddle River, NJ: Pearson Prentice Hall.

\section{AUTHOR BIOGRAPHIES}

MICHAEL FINDLAY is an instructor in the Department of Mathematical Sciences at the United States Military Academy, West Point, NY. He received a B.S. in Biomedical Engineering from Northwestern University in 1999, an M.S. in Operations Research from the Florida Institute of Technology in 2008, and an M.S. in Industrial Engineering from the University of Oklahoma in 2011. His research interests include applications of simulation and operations research to healthcare and military systems. His email addressism.findlay@us.army.mil.

HANK GRANT is the Director of the Center for Study of Wireless Electromagnetic Compatibility and Dugan Professor of Industrial Engineering at the University of Oklahoma. He holds B.S., M.S., and Ph.D. degrees in Industrial Engineering from Purdue University. Dr. Grant has held positions at the National Science Foundation and at Hewlett-Packard Labs. Dr. Grant was also involved in the startup and development of two Industrial Engineering software companies: Pritsker Corporation and FACTROL. His research interests include wireless electromagnetic compatibility, domain specific simulation languages, simulation and virtual reality, biomedical manufacturing, and order scheduling and promising. His email address ishgrant@ou.edu. 